\title{
IL-17 and Th17 cells in tuberculosis
}

\author{
Egídio Torrado and Andrea M. Cooper \\ Trudeau Institute, Inc., 154 Algonquin Ave., Saranac Lake, NY 12983
}

\begin{abstract}
Tuberculosis is primarily a disease of the lung. Constant expression of cellular immunity in this organ is required to control Mycobacterium tuberculosis growth, but this can also result in chronic inflammation and pathologic consequences. During primary tuberculosis both IFN- $\gamma$ and IL-17producing cells are induced; both are potent inflammatory cytokines capable of inducing expression of chemokines that promote cell recruitment and granuloma organization throughout infection. During the chronic phase, a balance between Th1 and Th17 responses needs to be achieved to control bacterial growth and limit immunopathology, as a shift of the response towards excessive IL-17 production may sustain extensive neutrophil recruitment and tissue damage. Thus, regulation of Th1 and Th17 responses during tuberculosis is essential to promote anti-mycobacterial immunity and prevent extensive immunopathological consequences.
\end{abstract}

\section{Keywords}

tuberculosis; lung; cytokines; inflammation; immunopathology

\section{Introduction}

Specialization of immune cells plays a central role in coordinating the immune response to different categories of pathogens. This specialization is typically best seen in CD4 T cell populations that produce discrete cytokines and display specific functions. For example, the control of pathogens that reside in phagocytes requires IFN- $\gamma$ secreting CD4 T cells to activate the anti-microbial mechanisms of the phagocyte; these cells are generally referred to as Thelper (Th) 1 in contrast with Th2 cells that produce IL-4, IL-5 and IL-13 and are important in controlling extracellular helminthes [1]. Recently, a new Th cell population has been identified and referred to as Th17. Th17 cells produce IL-17A (simply referred as IL-17) [2,3], IL-17F [4], IL-21 [5] and IL-22 [6] as their signature cytokines.

As the IL-17 receptor (IL-17RA) is ubiquitously expressed in different organs, including lung, liver and spleen, cells capable of responding to IL-17 include dendritic cells (DC) and macrophages, lymphocytes, epithelial cells, keratinocytes and fibroblasts [7,8]. IL-17induced responses include the expression of proinflammatory genes such as G-CSF, CXC chemokines, IL-6, IL-8 (MIP-2 in mice), antimicrobial proteins such as defensins and S100 proteins, granulopoiesis, neutrophil recruitment and inflammation [9]. Indeed, in infection models, IL-17 and Th17 cells were first implicated in the protective immune response to

\footnotetext{
(C) 2010 Elsevier Ltd. All rights reserved.
}

Corresponding author: acooper@trudeauinstitute.org, Tel: 518-891-3080, Fax: 518-891-5126.

Publisher's Disclaimer: This is a PDF file of an unedited manuscript that has been accepted for publication. As a service to our customers we are providing this early version of the manuscript. The manuscript will undergo copyediting, typesetting, and review of the resulting proof before it is published in its final citable form. Please note that during the production process errors may be discovered which could affect the content, and all legal disclaimers that apply to the journal pertain. 
rapidly growing extracellular bacteria in the lung and gut mucosal surfaces through efficient induction of neutrophil recruitment and tissue repair [10-12].

As this is a relatively newly identified subset, we are still learning about the complexity of Th17 cells and Th17-derived cytokines. Recent data suggests a broader and more complex role for these cells and cytokines in different infections including intracellular bacteria, fungi and virus at different mucosal surfaces [13]. Indeed, Th17 cells and Th17 derived cytokines were shown to be key inducers of inflammation and tissue damage in animal models of autoimmune diseases [14,15] and infection [13]. Therefore, the balance between Th17 mediated protection and pathology is key in defining the outcome of infections at the mucosa. Understanding this fine balance is central in developing new and improved preventive and therapeutic strategies.

The intracellular pathogen Mycobacterium tuberculosis (Mtb) causes the disease tuberculosis (TB), which kills over two million people every year [16]. It is thought that Th1 cells are important in the control of Mtb proliferation [17-21] however, despite vaccines having been developed to specifically potentiate this type of response none of these strategies have improved upon BCG, the currently used vaccine [22]. In order to improve BCG and generate more effective vaccination strategies, we need to more clearly understand what the components of the protective immune response are, and how they are regulated. The potential for IL-17 to mediate immune pathology as seen in autoimmune diseases and infection models, suggests that IL-17 may have detrimental effect in chronic bacterial infections such as TB. In order to spread and infect new hosts, Mtb is actually dependent on effective immunity and generation of granulomatous responses. Specifically in susceptible individuals, the granuloma develops to eventually become necrotic (caseating tubercles), allowing bacilli to become extracellular and able to be expelled out of the lungs upon coughing. How this pathological response is regulated is still not well understood, but a balance between protective and damaging immunity needs to be achieved to allow control of Mtb proliferation and reduce transmission and morbidity.

The capacity of Th17 cells, and their associated cytokines, to be important players in combating infections and maintaining homeostasis of mucosal surfaces contrasts with their ability to be involved in tissue destruction. It is therefore of critical importance to understand their role both in the protective and in the damaging responses to Mtb infection. To help in this regard we here discuss new advances in the understanding on the role of IL-17 and Th17 derived cytokines in the host immune response to Mtb, with emphasis in the induction and regulation of these responses and the mechanisms of protection and pathology associated with IL-17 and Th17 cells.

\section{Induction of IL-17 and Th17 responses in tuberculosis}

Following deposition of Mtb in the lung, it is likely that DCs migrate to the draining lymph nodes (dLN) where T cells are primed [23]. In the dLN, both IFN- $\gamma$ and IL-17 producing T cells are induced and these cells then migrate to the lung where they exert effector function. Although we are still learning how mycobacteria stimulate innate immune receptors, cytokines produced by Mtb-primed DCs are likely to be crucial for balanced acquired cellular responses [23].

The development of Th17 cells is initiated by IL- 6 or IL-21 in the presence of low amounts of TGF- $\beta$ [5,24-28]; IL-1 $\beta$ and TNF may also act as cofactors for Th17 differentiation $[29,30]$. The combinatory signal of these cytokines in newly primed $\mathrm{T}$ cells induces the expression of the orphan nuclear receptor ROR $\gamma \mathrm{t}$ via STAT-3. STAT-3 and ROR $\gamma \mathrm{t}$ induce the expression of the receptor for IL-23 resulting in the subsequent signaling of this cytokine which then stabilizes the Th17 phenotype [31]. While we have not studied the role of TGF- $\beta$ 
or IL-6 in the generation of Th17 cell in TB, we know that IL-23 is required for this type of response as mice deficient in IL-23p19 are unable to sustain Th17 responses and IL-17 mRNA expression throughout infection [32]. There is however an IL-17 response in the absence of IL-12p40 in the dLNs, but not in the lung [33,34], showing that upon Mtb infection, although IL-23 is not required for initial differentiation of IL-17-producing cells, expression of this cytokine in the infected organs is required to sustain IL-17 responses. On the other hand, development of Th1 responses is dependent upon IL-12p70 [35].

With the above set of criteria in mind it is clear that the relative levels of specific cytokines produced by mycobacterial infected cells, both at the site of induction and at the site of inflammation, are crucial for relative balance of Th1 and Th17 responses. In this regard the initiation of acquired immune responses in TB has been studied mostly by analyzing expression of IL-12p40 by antigen-presenting cells (APC) in response to Mtb. As this molecule is shared by both IL-12p70 and IL-23, understanding the pathways that lead to expression of IL-12p40, IL-12p70 and IL-23 are obviously important. One of the most important criteria in the induction of these cytokines is the pattern recognition receptors ligated on the APCs when they are exposed to the bacteria; Toll-Like receptors (TLR), NOD-like receptors and C-type lectins have all been implicated in this response (Figure 1).

TLR are known important inducers of IL-12p40 [36] and following low dose Mtb infection, TLR9 was shown to be required for IL-12p40 induction in vivo while both TLR2 and TLR9 could mediate IL-12p40 production by macrophages and DCs in response to live Mtb in vitro [37]. Interestingly, while mice deficient in either TLR2 or TLR9 were not significantly more susceptible to Mtb, mice lacking both molecules displayed markedly enhanced susceptibility to low dose aerosol Mtb infection [37]. Upon high dose challenge however, TLR9 deficiency was enough to increase susceptibility, suggesting that TLR2 alone is not able to compensate when bacterial burden is high [37]. These data suggest that a crosstalk between TLRs is critical to induce IL-12p40 expression and generate efficient acquired immunity (Figure 1).

The intracellular NOD-like receptor NOD2/CARD15 has also been implicated in the recognition of Mtb [38,39]. Indeed, Mycolylarabinogalactan-peptidoglycan, an Mtb cell wall component, was shown to stimulate macrophages and DCs to release IL-12p40 in a partially NOD2-dependent manner [39]. Although NOD2 deficient mice have similar bacterial burdens in the early stages of infection $[38,39]$, they have decreased inflammatory responses [38]. At six months post-infection, NOD2 deficient mice have increased bacterial burdens when compared with wild-type mice [38]. In humans, NOD2 variants were demonstrated to be associated with susceptibility to TB in African American populations [40].

Another major receptor involved in IL-12p40 induction is the C-type lectin Dectin-1. Although this receptor plays a major role in sensing fungal $\beta-1,3$ glucan linked carbohydrates, it has been shown that Dectin-1 plays a major role in promoting IL-12p40 production by mycobacterial infected splenic DCs [41]. Indeed, while TLR2 deficiency partially reduced IL-12p40 production, blockade of Dectin-1 in TLR2 deficient splenic dendritic cells further reduces IL-12p40 production suggesting cooperation between these receptors in IL-12p40 induction [41] (figure 1).

Collectively, these studies show that Mtb induces IL-12p40 expression through signals received upon ligation of different pathogen recognition receptors (Figure 1). During the course of infection, the balance in the secretion of bioactive IL-12p70 and IL-23 is achieved by the relative expression of IL-23p19 and IL-12p35 subunits to form the heterodimeric cytokines. The expression of these subunits is not so well understood during TB, but it is likely that besides pathogen recognition receptors signaling, further cytokine signaling in the 
cytokine-producing cell is important. A recent analysis of the response of human DCs to Mtb showed that IL-23 was preferentially produced, likely in a TLR2 dependent manner, compared to IL-12p70 in the absence of IFN- $\gamma$ signaling. Following IFN- $\gamma$ activation, Mtb stimulated DCs secreted both IL-12 and IL-23 [30] (figure 1). These data suggest that before IFN- $\gamma$ expression, the preferential T cell effector type is an IL-17 producer, but later during infection both IFN- $\gamma$ and IL-17 are induced.

The use of defined adjuvants that target pathogen recognition receptors on APCs to induce specific subsets of cells is essential to induce specific cellular responses by vaccination. In this respect, complete Freund's adjuvant (CFA) has been used for a long time in research as it is a potent inducer of cell-mediated inflammatory responses. Since heat killed Mtb is a major component of CFA, understanding how specific elements of this adjuvant target cells and stimulate inflammation will help in adjuvant design. Recent exciting work has defined the pathway by which one of the major components of Mtb cell wall, Trehalose-6,6'dimycolate (TDM; Cord factor) induces cell-mediated inflammatory responses [42-44]. These results showed that the C-type lectin Mincle is central for TDM to induce inflammatory responses and both Th1 and Th17 acquired immunity [42,43]. Indeed, Trehalose-6,6-dibehenate (a synthetic analogue of TDM) and TDM stimulation of macrophages were shown to induce IL-12p40, IL-12p70, IL-23, IL-1 $\beta$, IL-6 and TNF production in a MyD88-independent way but dependent on Mincle signaling through SykFcR $\gamma$-CARD9 [42-44]. Interestingly, it has been suggested that $\mathrm{T}$ cells can also respond to TDM as Mincle is expressed in T cells upon activation [42]. It is possible that direct T cell recognition of TDM induces production of cytokines such as IFN- $\gamma$, IL-17 or IL-1 $\beta$. In line with this, it has been shown that direct T cell stimulation through TLR2 promotes Th17 differentiation in vitro and more robust Th17 cytokine production [45].

Together, these data show that Mtb is recognized by distinct host receptors and is able to induce both Th1 and Th17 differentiating cytokines. While signaling through diverse pathogen recognition receptors promotes IL-12p70 and IL-23, further cytokine signaling balances this production. Understanding how these signals act on immune cells is central to developing defined adjuvants that promote specific responses. It is also important to further understand what comprises protective immunity and how is this immunity regulated.

\section{The protective immune response to primary Mycobacterium tuberculosis infection: do IL-17 and Th17 derived cytokines play a role?}

As discussed above, early studies using respiratory infection models, established a central role for IL-17 in the protective immune response to rapidly growing extracellular bacterial pathogens, mediated by efficient neutrophil recruitment and tissue repair [10-12]. In intracellular bacterial infections however, the role of IL-17 is not so well understood. Recent studies suggest that IL-17 enhances immunity against some intracellular pathogens; however the effect is not as dramatic as for extracellular bacterial infections. For example, IL-17 deficient mice have increased bacterial burdens in the spleen and liver following infection with a sublethal dose of Salmonella enterica [46]. Similarly, after Listeria monocytogenes infection, increased bacterial burdens and defective granuloma organization are observed in the liver [47]. In both models defective neutrophil recruitment was associated with the enhanced susceptibility of IL-17 deficient mice $[46,47]$. Whether this is the only mechanism of by which IL-17 enhances immunity to these pathogens is still not known.

As human studies are naturally restricted, we can more thoroughly investigate the role of IL-17 and Th17-derived cytokines in the mouse model, as their function may be dependent on the stage of infection or the bacterial burden. Indeed, while the absence of IL-23 or IL-17 signaling does not significantly impact the ability of mice to control Mtb following a low 
dose aerosol infection [32,48], after high dose intratracheal infection, mice deficient in IL-17 are unable to control Mtb [49]. The $\gamma \delta \mathrm{T}$ cell population is a major source of early IL-17 during mycobacterial infections [50,51], specially upon high dose infection [47]. Indeed, upon intratracheal delivery of BCG, IL-17 mRNA was detected in the lungs of infected animals as early as one day after infection [51] and $\gamma \delta \mathrm{T}$ cells expressing the $\mathrm{V} \gamma 4$ or $\mathrm{V} \gamma 6$ TCR chains were identified as the primary source of IL-17 [47]. Following low dose aerosol infection with Mtb, $\gamma \delta$ T cells are also an important source of IL-17 [50] and this response is mostly antigen-independent but dependent upon IL-23, as supernatants from Mtb-infected DCs that contain IL-23 could induce IL-17 secretion from $\gamma \delta$ T cells purified from both naïve and Mtb infected mice [50]. In line with these data, $\gamma \delta$ T cells were reported to produce IL-17, IL-21 and IL-22 in response to IL-1 $\beta$ and IL-23 without TCR engagement and act in an amplifying loop for IL-17 production by Th17 cells [52]. It is likely that also during TB, $\gamma \delta$ T-cell-derived IL-17 act to amplify IL-17 production by Th7 cells.

Together these data suggest that $\gamma \delta$ T cells are an important early source of IL-17 during mycobacterial infections. Further studies are required to address the impact of the bacterial dose and route of infection in the induction of IL-17 by $\gamma \delta \mathrm{T}$ cells and the subsequent role of these cells and cytokine role in expression of protective and immunopathologic consequences in TB.

As IL-17 is mostly associated with induction of proinflammatory programs associated with chemokine secretion and neutrophil recruitment, it is not surprising that absence of this cytokine does not significantly impact the ability of mice to control Mtb, which requires accumulation of mononuclear cells. However, the presence of this cytokine may facilitate recruitment of protective cells and organization of cellular foci in the lung. In this respect, following Mtb infection, the early recruitment of neutrophils to Mtb lungs is associated with early granuloma formation [53]. In addition, neutrophils and macrophages cooperate for efficient mycobacterial killing [54] (Figure 2).

Both IL-23 and IL-17 can affect neutrophil homeostasis and survival [55,56]. In the absence of IL-17, neutrophils are found in reduced numbers after BCG infection [51] or when IL-17 is neutralized following aerosol Mtb infection [57]. Promoting neutrophil recruitment and survival is probably one of the mechanisms by which the IL-23/Th17 axis promotes granuloma integrity independently of the protective response (figure 2). Indeed, during the acute phase of infection, neutrophils and macrophages are the predominant hematopoietic cell types followed by a decrease in the number of neutrophils during the chronic phase [58]. Neutralization of neutrophils during Mtb infection was shown to delay granuloma formation in mice with no influence on bacterial loads [53]. Neutrophil-mediated regulation of granuloma formation was shown to be in part dependent on chemokines signaling through CXCR3, in particular CXCL9 as neutrophil neutralization ablated expression of this chemokine [53]. In accordance, neutrophils were the main source of CXCL9 early after infection and antibody blockade of this chemokine resulted in the same deficient granuloma development as neutrophil neutralization [53].

As discussed above, $\gamma \delta \mathrm{T}$ cells are an important source of IL-17 upon high dose intratracheal BCG infection and low dose Mtb aerosol infection. In the BCG model, both IL-17 and $\gamma \delta$ T cell deficient mice show delayed granuloma formation, suggesting that $\gamma \delta \mathrm{T}$ cell-derived IL-17 is essential for initial granuloma organization, probably by promoting early neutrophil recruitment (figure 2). However, unlike in the BCG model, low dose aerosol Mtb infected $\gamma \delta$ $\mathrm{T}$ cell deficient mice display enhanced cellular foci with elevated accumulation of neutrophils $[53,59]$. Antibody neutralization of neutrophils in these mice reduces this exacerbated accumulation of cells [53] further supporting the hypothesis that neutrophil accumulation promotes granuloma formation in the presence or absence of $\gamma \delta \mathrm{T}$ cells. Taken 
together, these data suggest a differential role for IL-17 producing $\gamma \delta \mathrm{T}$ cells in the low dose aerosol Mtb infection model versus the high dose intratracheal BCG model. Although initial cellular foci organization seem to be promoted by early neutrophil migration further research is required to understand the role of $\gamma \delta \mathrm{T}$ cells in this process.

Another potential mechanism of protection mediated by IL-17 against intracellular infections was recently identified [8]. Upon Francisella tularensis infection, the IL-23/Th17 axis was shown to enhance protection by promoting IL-12 secretion by DCs, therefore enhancing Th1 responses [8]. This effect was shown to be directly mediated by IL-17 signaling, as culture of $F$. tularensis-stimulated DCs in the presence of IL-17, but not IL-17F or IL-22, induced a 10 fold increase in IL-12 levels. Moreover, differentiation of naïve cells by $F$. tularensis-stimulated DCs into IFN- $\gamma$ producing cells was more efficient when IL-17 was added to the culture as compared to stimulated DCs alone [8]. Altogether, these data show a novel role for IL-17 in intracellular infections. This novel IL-17 function is yet to be examined in TB however, since Mtb is known to induce a strong IL-12 response [60] IL-17 may be dispensable. Therefore, it is not surprising the observation that IL-17RA deficient mice are not more susceptible to low aerosol infection [48]. Following high dose infection however, as IL-17 deficient are more susceptible to Mtb [49], it is possible that IL-17 may act to enhance the protective immune response by augmenting IL-12 secretion (Figure 2).

It has been shown that neutralization of neutrophils during the first week following Mtb intravenous infection enhances the susceptibility of mice, as a consequence of reduced expression of IFN- $\gamma$ in the infected organs [61]. When recombinant IL-12 was administered a reduction in the exacerbation of infection was observed in the livers of neutropenic mice [61], suggesting that the reduced IFN- $\gamma$ expression was caused by a reduced IL-12 production. As neutrophil recruitment can be promoted by IL-17, it is possible that early accumulation of neutrophils can also stimulate the differentiation of Th1 responses by promoting release of IL-12. Indeed, proteins released by neutrophil degranulation have been shown to promote IL-12 secretion by macrophages [62].

Further complexity in the role to IL-17 and Th17 responses in TB may be associated with the Th17 related cytokine IL-22. IL-22 acts through the IL-22 receptor complex expressed by epithelial cells and hepatocytes, where it promotes regeneration and protects against tissue damage $[63,64]$. In vivo and in vitro differentiation of Th17 cells leads to the development of cells expressing only IL-17, IL-22 and cells that coexpress both cytokines [6], suggesting that IL-22 production is not restricted to Th17 cells but most likely induced by Th17 differentiation factors. Indeed, in healthy humans exposed to mycobacteria, IL-22expressing CD4 T cells were reported as being distinct from Th17 and Th1 cells [65]. Interestingly, while IL-17 protein was not found in BAL fluid of TB patients, IL-22 was present [65], suggesting that IL-17 and IL-22 may have distinct roles in TB infection and disease. In a recent study in the TB mouse model, neutralization of IL-22 did not have any effect in the lung bacterial burden [66]. As IL-17 and IL-22 may be produced and act independently of each other, their redundancy needs to be determined, since the absence of one may be compensated by the other.

The data are equivocal with regard to whether IL-17 is required for protection against Mtb; in some hands it is required for control of bacteria and in others it is not; it is likely however that dose and route of infection are key in this issue. It is also important however to determine whether IL-17 plays a role in granuloma organization by promoting early neutrophil recruitment. More studies are required to further dissect the pathways by which IL-17 and Th17 related cytokines act to enhance cellular immune responses and cellular foci organization (Figure 2). 


\section{Role of IL-17 in tuberculosis mediated immunopathology}

Regulation of immunopathology during chronic Mtb infection is essential for host survival. As immunopathology is a central feature of Mtb lung infection, it is not surprising that IL-17 and Th17 cells have a role to play. Importantly, this role also seems to be mediated, at least in part, by neutrophils (Figure 2).

As discussed earlier, neutrophil recruitment and survival may be one of the mechanisms by which IL-17 promotes granuloma organization. However, this response needs regulation since the accumulation of large numbers of neutrophils in the lung is generally associated with a bad prognosis and increased lung pathology. Indeed, neutrophil accumulation is common in genetically susceptible mice [67,68]. In humans, it has been shown that neutrophils are the predominant infected cell types in active TB patients, suggesting that these cells provide a permissive environment for bacillary replication [69]. Interestingly, it was recently shown that a transcriptional signature in blood neutrophils, indicating activation of these cells with type I IFN- $\alpha \beta$ and IFN- $\gamma$ is present in patients with active as opposed to latent TB [70]. These data implicate the phenotype of neutrophils in the pathogenesis of TB. In line with these data it has also been shown that, exposure of neutrophils to IL-17 or IL-23 can change the homeostasis and phenotype of these cells making them more prone to cause immunopathology [56]. In other words, IL-17 may have beneficial effects early on, by recruiting neutrophils and promote secretion of tissue-specific homing chemokines, but extensive exposure of neutrophils to IL-17 or IL-23 can make these cells survive longer, change their phenotype and cause immunopathology (Figure 2).

The discrimination between protective and damaging immune responses during TB is extremely important for rational vaccine design. As BCG vaccination has variable efficacy [71] and lasts for a limited amount of time [72,73], revaccination with BCG or modified BCG strains, DNA vaccines or sub-unit vaccines are some potential strategies. We have recently shown that repetitive subcutaneous BCG vaccination in Mtb-infected mice results in an enhanced IL-17 response when compared to control mice. With the increase in IL-17 producing cells, elevated expression of the proinflammatory cytokines TNF, IL-6, and MIP-2 also occurred concurrently with an enhanced influx of neutrophils to the lungs [74]. Importantly, this response is highly pathological causing extensive tissue damage without changing the protective immune response. The enhanced accumulation of IL-17-producing cells in the lung is not a result of differentiation of these cells in the dLN, but it is likely dependent on IL-23 expression locally and IL-23p19 deficient mice or IL-17 antibody blockade in this mode, prevented neutrophil accumulation and the increased pathology [74] (Figure 2). Interestingly, as the number of IFN- $\gamma$ producing cell was not affected by repetitive BCG vaccinations in the lungs, it appears that the IL-17 response became refractory to regulation by the IFN- $\gamma$ response [74]. This is surprising, as IFN- $\gamma$ responses can regulate IL-17 responses following BCG infection [75].

These data show that re-exposure of Mtb-infected mice to high antigen challenge, promotes further expansion of an ongoing Th17 response which causes extensive lung pathology associated with elevated recruitment of neutrophils. Importantly, this enhanced Th17 response is supported locally by elevated expression of IL-23 and does not affect the protective immune response (Figure 2).

In line with these observations, it was recently demonstrated that IFN- $\gamma$ signaling by lung stromal cells is critical to regulate immunopathology following Mtb aerosol infection [76]. In this study, chimeric mice with IFN- $\gamma$-unresponsive lung stromal cells exhibit early mortality and increased Mtb bacterial burdens when compared with control mice. This response was caused by impaired expression of Indoleamine 2,3-dioxygenase (IDO) by lung 
epithelial and endothelial cells, overexpression of IL-17 and enhanced neutrophilic infiltration in the lungs [76]. Also here, the enhanced IL-17 response was associated with an elevated expression of IL-23 as well as IL- 6 and TGF- $\beta$ in the lungs of infected animals [76]. Importantly, impaired IDO expression causing a reduced catabolism of tryptophan into kynurenines was shown to be the mechanism behind the unrestrained IL-17 response. Indeed, tryptophan catabolic products inhibited IL-23 dependent IL-17-production by in vitro differentiated Th17 cells. Although inhibition of IFN- $\gamma$ by tryptophan catabolites in CD4 T cells during Th1 differentiation was also observed, the concentrations required were substantially higher than those required for Th17 inhibition [76]. In addition to reduced IDO expression, it is possible that in this model the increased antigen availability due to elevated bacterial burdens in chimeric mice may also contribute to the elevated IL-23 expression and therefore, both reduced IDO and the elevated IL-23 expression could contribute to the enhanced IL-17 response.

Overall these data show that, as for the nonpathogenic IL-17 response to mycobacterial infection, IL-23 expression in the infection site is critical for maintenance of pathogenic IL-17 responses. Moreover, repetitive antigen stimulation of IL-17 producing cell and elevated expression of IL-23 at the site of infection further expands this population and shifts the balance of the response towards an elevated IL-17 production, extensive numbers of neutrophils infiltrate the lungs and cause tissue damage. Therefore, regulation of these responses is key for host survival. In this respect, IFN- $\gamma$ appears to be a key regulator of IL-17 responses in mycobacterial infections, both directly in the differentiation of IL-17 producing cells [75] and indirectly by inducing release of tryptophan catabolic products through IDO activation by epithelial and endothelial cells [76].

While IL-23 expression at the site of infection is important for survival of IL-17 producing cells, another member of the IL-12 family, IL-27, has been shown to inhibit this type of response. Indeed, IL-27 signaling was shown to be an important inhibitory cytokine for Th17 differentiation and limits inflammation of autoimmune [77,78] and infectious origin [79]. Although the role of this cytokine is still not well understood in TB, two different studies have shown that IL-27R signaling has detrimental effects for the control of Mtb in the mouse model [80,81]. Indeed, IL-27R deficient mice have lower Mtb bacterial burdens in comparison to wild-type mice however, this deficiency is associated with decreased survival and enhanced granulomatous responses [81]. Whether this is caused by an enhanced IL-17 response remains to be answered. Besides regulating IL-17/Th17 type of responses, IL-27 has been shown to induce IL-10 production from activated CD4 T cells [82]. IL-10 deficient mice were recently shown to have an elevated production of chemokines and cytokines including IL-17, an accelerated and enhanced accumulation of IFN- $\gamma$ producing cells in the lung and are more resistant to Mtb infection. Although antibody neutralization of IL-17 does not significantly affect the accumulation of IFN- $\gamma$ producing T cells in the lungs, it reduces the numbers of neutrophils and bacterial loads in the spleen of Mtb-infected mice [57].

These data suggest a role for IL-10 in the regulation of IL-17 responses to TB as it has been shown in other infection models [83]. In addition, IL-27 inhibition of Th17 differentiation as well as by inducing IL-10 production by activated CD4 T cells may function to suppress excessive T cell activation. Therefore, both IL-27 and IL-10 can serve as a counterregulatory cytokines to prevent extensive immunopathology by keeping cellular responses in check. 


\section{Cellular regulation of IL-17 dependent immunopathology in tuberculosis}

As discussed, TGF- $\beta$ is a central cytokine in the differentiation of Th17 cells; however this cytokine also acts in the differentiation of Foxp3-expressing Treg cells [84]. It has been shown that foxp3-expressing Treg cells expand during experimental Mtb infection and upon deletion Mtb bacterial burdens decrease [85]. In humans, there are more Treg cells in active TB patients than patients with latent infection [86] or in healthy tuberculin responders [87]. Overall these data suggest a largely negative role for Treg cells in TB; however their presence in the infected organs may be important for balancing protective and damaging immune responses.

As both Th17 and Treg cells are present during Mtb infection it is important to understand what regulates the relative levels of each population. TGF- $\beta$ induces expression of foxp3 but further signaling by STAT3-activating cytokines such as IL-6 promotes Th17 differentiation [24,25,84]. As Mtb induces early secretion of both IL-6 and IL-23, Th17 development is promoted, while Treg cells expand from a population of preexisting foxp3-expressing cells [88]. It is still not clear what factors promote expansion of Treg cells during TB; however, the relative levels of ROR $\gamma \mathrm{t}$ and foxp3 expression as determined by the amount of TGF- $\beta$ and STAT3 signaling cytokines will certainly be important for the relative levels of Th17 and Treg cells. Therefore, during inflammatory responses where IL-23 and IL-6 are highly expressed, Treg cells may not expand efficiently and inflammation may develop without regulation. Further research is required to clarify this subject. In addition, recent data suggest an increased plasticity in Treg cells and Th17 cell; indeed, Treg cells can convert into IL-17 producers by pathogen components or inflammatory mediators, such as IL-6 [89]. Treg conversion into IL-17 producers is yet to be analyzed in TB, but it may be of paramount importance in the regulation of inflammation and control of bacillary growth, as it is possible that sustained expression of IL- 6 and IL-23 in the lungs of infected animals promote conversion of Treg cells into IL-17 producers (figure 2).

Recent data link the transcription factor T-bet, important in Th1 differentiation, with the function of Treg cells during Mtb infection and in the absence of this transcription factor Treg cells fail to persist [90]. It is interesting that T-bet deficient mice, although more susceptible to Mtb when compared to wild-type mice, do not succumb to infection with the same kinetics as IFN- $\gamma$ deficient mice [91] probably because T cells from Mtb infected T-bet deficient mice retain some capacity to produce IFN- $\gamma$ following in vitro stimulation [91]. As Mtb is a strong inducer of type 1 immunity as well as IL-17-producing cells, it is possible that the absence of T-bet shifts the response to IL-17 production. Indeed, T-bet deficient mice infected with Mtb have severe inflammation as might be expected of an environment with high IL-17-producing cells [91]. Similarly, in Trypanosoma cruzi infection it has been shown that T-bet deficient CD4 T cells develop normally and produce similar levels of IFN$\gamma$ as wild-type cells however, a robust IL-17 response develops [92]. Thus, microenvironmental factors may impair T-bet expression on a per cell basis, not significantly limiting IFN- $\gamma$ production per cell but enough to impact T-bet inhibition of ROR $\gamma \mathrm{t}$ and production of IL-17. It will be interesting to determine whether T-bet expression levels have any effect in IL-17 production by IFN- $\gamma$-producing cells upon Mtb infection, and to what extent these cells are relevant to control infection or to cause immunopathological consequences. In this respect, IL-27 has been shown to induce T-bet expression in CD4 T cells [93] and IL-27R have decreased survival and enhanced inflammation when compared to wild-type mice [80]. It will be intriguing to determine whether this is caused by lower Tbet expression and to what extent does this affect differentiation of IL-17-producing cells. 


\section{Conclusion}

TB remains one of the leading causes of death from a single infectious agent worldwide. In order to generate better vaccination strategies we need to further define the protective and pathological mechanisms of the immune response to Mtb. While the IL-23/IL-17 axis does not seem to be essential for primary control of Mtb, recent data suggests a critical role of this axis in vaccine induced protection. In part, this effect is mediated by the release of chemokine gradients that recruit protective cells to the infection foci. However, there is a down side to this effect. Excessive production of IL-23 and IL-17 causes immunopathology due to excessive recruitment and phenotypic change of inflammatory cells. In this respect, the fine balance between Th1 and Th17 responses is central in defining the outcome of Mtb infection. It is therefore critical to further elucidate the mechanisms of protection and pathology associated with Th17 and IL-17-producing cells and use that knowledge in our advantage to generate rational vaccination strategies that promote balanced acquired responses with minimal collateral damage.

\section{Acknowledgments}

Our work is supported by the Trudeau Institute, Inc., NIH grants AI46530, AI67723, AI69121 and an American Lung Association De Souza award.

\section{Biography}

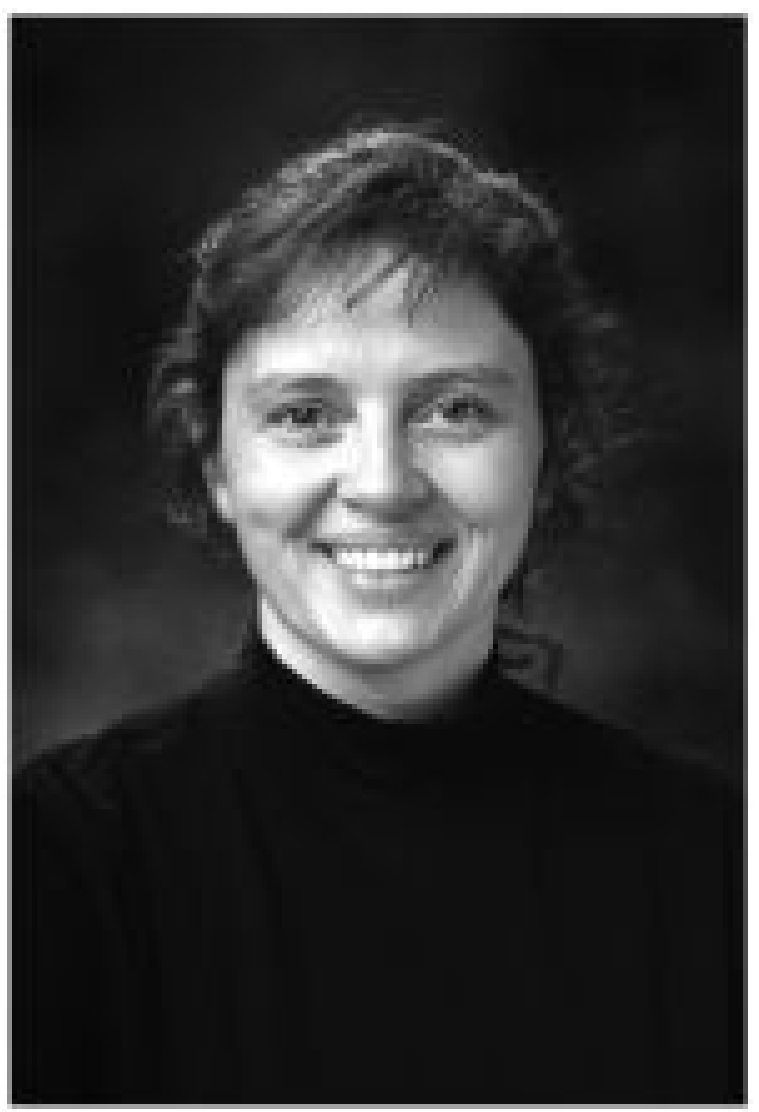

Dr. Andrea Cooper began her scientific career at The London School of Hygiene and Tropical Medicine where she helped describe the interaction between macrophages and protozoan parasites of the genus Leishmania. She then moved to the National Institutes of 
Health in Bethesda, Maryland, and expanded her investigation of leishmaniasis and leishmanial antigens to include the T-cell-mediated response of patients suffering from cutaneous, mucocutaneous and visceral forms of this disease. She then moved to the Mycobacterial Research Labs, Colorado State University and began studying the protective immune response to Mycobacterium tuberculosis. At Colorado State, Dr. Cooper elucidated the essential role of the Interferon-gamma [IFN-y]-Interleukin-12 [IL-12] pathway in protection from mycobacterial disease. Dr. Cooper then moved to the Trudeau Institute. This allowed her to focus her investigation on the cellular immune response to Mycobacterium tuberculosis. Recent work has resulted in the definition of the roles of IL-12, IL-23 and IL-27 in both the primary and vaccine-induced immune response to this pathogen in the lung.

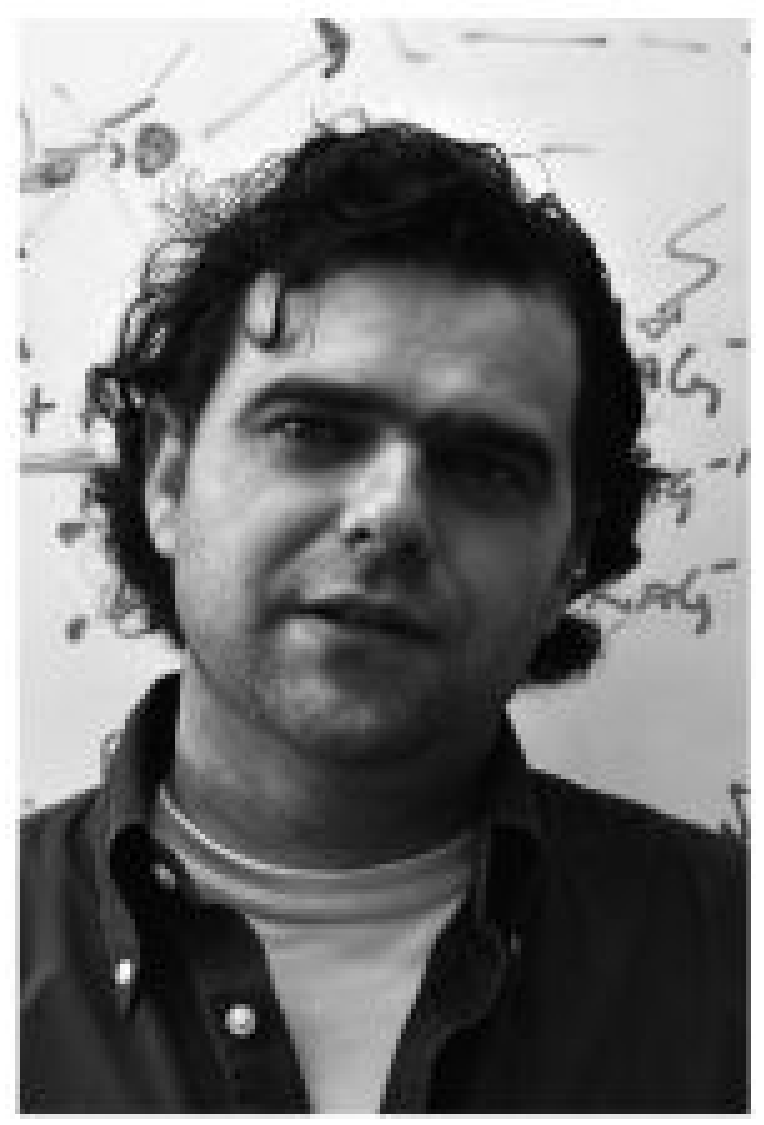

Egidio Torrado earned his bachelor's degree in Biology from University of Aveiro in Portugal. During his graduate studies at the Life and Health sciences Research Institute, University of Minho in Dr. Jorge Pedrosa's laboratory, he investigated the interaction of Mycobacterium ulcerans and the effects of mycolactone in the activation of host phagocytes. He is currently a post-doctoral fellow in Dr. Andrea Cooper's laboratory at the Trudeau Institute in Saranac Lake where his research is aimed at better understanding the expression and regulation of the immune response to Mycobacterium tuberculosis in the lung.

\section{References}

[1]. Laurence A, O'Shea JJ. T(H)-17 differentiation: of mice and men. Nat Immunol 2007;8:903-5. [PubMed: 17712339] 
[2]. Harrington L, Hatton R, PR M, Turner H, Murphy T, KM M, et al. Interleukin-17 producing $\mathrm{CD} 4(+)$ effector $\mathrm{T}$ cells develop via a lineage distinct from the T helper 1 and 2 lineages. Nat Immunol 2005;6:1123-32. [PubMed: 16200070]

[3]. Park H, Li Z, Yang X, SH C, Nurieva R, Wang Y, et al. A distinct lineage of CD4 T cells regulates tissue inflammation by producing interleukin-17. Nat Immunol 2005;6:1133-41. [PubMed: 16200068]

[4]. Langrish C, Chen Y, Blumenschein W, Mattson J, Basham B, Sedgwick J, et al. IL-23 drives a pathogenic T cell population that induces autoimmune inflammation. J Exp Med 2005;201:23340. [PubMed: 15657292]

[5]. Korn T, Bettelli E, Gao W, Awasthi A, Jäger A, Strom T, et al. IL-21 initiates an alternative pathway to induce proinflammatory $\mathrm{T}(\mathrm{H}) 17$ cells. Nature 2007;448:484-87. [PubMed: 17581588]

[6]. Liang S, Tan X, Luxenberg D, Karim R, Dunussi-Joannopoulos K, Collins M, et al. Interleukin (IL)-22 and IL-17 are coexpressed by Th17 cells and cooperatively enhance expression of antimicrobial peptides. J Exp Med 2006;203:2271-79. [PubMed: 16982811]

[7]. Yao Z, Fanslow WC, Seldin MF, Rousseau AM, Painter SL, Comeau MR, et al. Herpesvirus Saimiri encodes a new cytokine, IL-17, which binds to a novel cytokine receptor. Immunity 1995;3:811-21. [PubMed: 8777726]

[8]. Lin Y, Ritchea S, Logar A, Slight S, Messmer M, Rangel-Moreno J, et al. Interleukin-17 is required for $\mathrm{T}$ helper 1 cell immunity and host resistance to the intracellular pathogen Francisella tularensis. Immunity 2009;31:799-810. [PubMed: 19853481]

[9]. Ouyang W, Kolls JK, Zheng Y. The biological functions of T helper 17 cell effector cytokines in inflammation. Immunity 2008;28:454-67. [PubMed: 18400188]

[10]. Ye P, Garvey PB, Zhang P, Nelson S, Bagby G, Summer WR, et al. Interleukin-17 and lung host defense against Klebsiella pneumoniae infection. Am J Respir Crit Care Med 2001;25:335-40.

[11]. Ye P, Rodriguez FH, Kanaly S, Stocking KL, Schurr J, Schwarzenberger P, et al. Requirement of Interleukin-17 receptor signalling for lung CXC chemokine and granulocyte colony-stimulating factor expression, neutrophil recriutment, and host defense. J Exp Med 2001;194:519-27. [PubMed: 11514607]

[12]. Happel K, Dubin P, Zheng M, Ghilardi N, Lockhart C, Quinton L, et al. Divergent roles of IL-23 and IL-12 in host defense against Klebsiella pneumoniae. J Exp Med 2005;202:761-69. [PubMed: 16157683]

[13]. Khader SA, Gaffen SL, Kolls JK. Th17 cells at the crossroads of innate and adaptive immunity against infectious diseases at the mucosa. Mucosal Immunol 2009;2:403-11. [PubMed: 19587639]

[14]. Dong C. Regulation and pro-inflammatory function of interleukin-17 family cytokines. Immunol Rev 2008;226:80-6. [PubMed: 19161417]

[15]. Diveu C, McGeachy MJ, Cua DJ. Cytokines that regulate autoimmunity. Curr Op Immunol 2008;20:663-8.

[16]. Maartens G, Wilkinson RJ. Tuberculosis. Lancet 2007;370:2030-43. [PubMed: 17719083]

[17]. Cooper AM, Dalton DK, Stewart TA, Griffin JP, Russell DG, Orme IM. Disseminated tuberculosis in interferon gamma gene-disrupted mice. J Exp Med 1993;178:2243-7. [PubMed: 8245795]

[18]. Cooper AM, Roberts AD, Rhoades ER, Callahan JE, Getzy DM, Orme IM. The role of interleukin-12 in acquired immunity to Mycobacterium tuberculosis infection. Immunology 1995;84:423-32. [PubMed: 7751026]

[19]. Cooper AM, Magram J, Ferrante J, Orme IM. IL-12 is crucial to the development of protective immunity in mice intravenously infected with Mycobacterium tuberculosis. J Exp Med 1997;186:39-46. [PubMed: 9206995]

[20]. Pearl JE, Saunders BM, Ehlers S, Orme IM, Cooper AM. Inflammation and lymphocyte activation during mycobacterial infection in the interferon-g-deficient mouse. Cell Immunol 2001;211:43-50. [PubMed: 11585387] 
[21]. Cooper AM, Kipnis A, Turner J, Magram J, Ferrante J, Orme IM. Mice lacking bioactive IL-12 can generate protective, antigen-specific cellular responses to mycobacterial infection only if the IL-12 p40 subunit is present. J Immunol 2002;168:1322-27. [PubMed: 11801672]

[22]. Kaufmann SH. Novel tuberculosis vaccination strategies based on understanding the immune response. J Intern Med 2010;267:337-53. [PubMed: 20433580]

[23]. Cooper A. Cell mediated immune responses in tuberculosis. Ann Rev Immunol 2009;27:393422. [PubMed: 19302046]

[24]. Veldhoen M, Hocking R, Atkins C, Locksley R, Stockinger B. TGFbeta in the context of an inflammatory cytokine milieu supports de novo differentiation of IL-17-producing T cells. Immunity 2006;24:179-89. [PubMed: 16473830]

[25]. Bettelli E, Carrier Y, Gao W, Korn T, Strom T, Oukka M, et al. Reciprocal developmental pathways for the generation of pathogenic effector TH17 and regulatory T cells. Nature 2006;441:235-38. [PubMed: 16648838]

[26]. Mangan P, Harrington L, O'Quinn D, Helms W, Bullard D, Elson C, et al. Transforming growth factor-beta induces development of the $\mathrm{T}(\mathrm{H}) 17$ lineage. Nature 2006;441:231-34. [PubMed: 16648837]

[27]. Zhou L, Ivanov I, Spolski R, Min R, Shenderov K, Egawa T, et al. IL-6 programs T(H)-17 cell differentiation by promoting sequential engagement of the IL-21 and IL-23 pathways. Nat Immunol 2007;8:967-74. [PubMed: 17581537]

[28]. Nurieva R, Yang X, Martinez G, Zhang Y, Panopoulos A, Ma L, et al. Essential autocrine regulation by IL-21 in the generation of inflammatory T cells. Nature 2007;448:480-83. [PubMed: 17581589]

[29]. van Beelen AJ, Zelinkova Z, Taanman-Kueter EW, Muller FJ, Hommes DW, Zaat SA, et al. Stimulation of the intracellular bacterial sensor NOD2 programs dendritic cells to promote interleukin-17 production in human memory T cells. Immunity 2007;27:660-9. [PubMed: 17919942]

[30]. Gerosa F, Baldani-Guerra B, Lyakh L, Batoni G, Esin S, Winkler-Pickett R, et al. Differential regulation of interleukin 12 and interleukin 23 production in human dendritic cells. J Exp Med 2008;205:1447-61. [PubMed: 18490488]

[31]. Zhu J, Yamane H, Paul WE. Differentiation of effector CD4 T cell populations. Ann Rev Immunol 2010;28:445-89. [PubMed: 20192806]

[32]. Khader S, Pearl J, Sakamoto K, Gilmartin L, Bell G, Jelley-Gibbs D, et al. IL-23 compensates for the absence of IL-12p70 and is essential for the IL-17 response during tuberculosis but is dispensable for protection and antigen-specific IFN-gamma responses if IL-12p70 is available. $\mathrm{J}$ Immunol 2005;175:788-95. [PubMed: 16002675]

[33]. Wozniak T, Ryan A, Britton W. Interleukin-23 restores immunity to Mycobacterium tuberculosis infection in IL-12p40-deficient mice and is not required for the development of IL-17-secreting T cell responses. J Immunol 2006;177:8684-92. [PubMed: 17142769]

[34]. Khader S, Bell G, Pearl J, Fountain J, Rangel-Moreno J, Cilley G, et al. IL-23 and IL-17 in establishment of protective pulmonary CD4+ T cell responses upon vaccination and during Mycobacterium tuberculosis challenge. Nat Immunol 2007;8:369-77. [PubMed: 17351619]

[35]. Cooper A, Solache A, Khader S. Interleukin-12 and tuberculosis: An old story revisited. Curr Op Immunol 2007;19:441-47.

[36]. Goriely S, Neurath M, Goldman M. How microorganisms tip the balance between interleukin-12 family members. Nat Rev Immunol 2008;8:81-86. [PubMed: 18084185]

[37]. Bafica A, Scanga C, Feng C, Leifer C, Cheever A, Sher A. TLR9 regulates Th1 responses and cooperates with TLR2 in mediating optimal resistance to Mycobacterium tuberculosis. J Exp Med 2005;202:1715-24. [PubMed: 16365150]

[38]. Divangahi M, Mostowy S, Coulombe F, Kozak R, Guillot L, Veyrier F, et al. NOD2-deficient mice have impaired resistance to Mycobacterium tuberculosis infection through defective innate and adaptive immunity. J Immunol 2008;181:7157-65. [PubMed: 18981137]

[39]. Gandotra S, Jang S, Murray PJ, Salgame P, Ehrt S. Nucleotide-binding oligomerization domain protein 2-deficient mice control infection with Mycobacterium tuberculosis. Infect Immun 2007;75:5127-34. [PubMed: 17709422] 
[40]. Austin CM, Ma X, Graviss EA. Common nonsynonymous polymorphisms in the NOD2 gene are associated with resistance or susceptibility to tuberculosis disease in African Americans. J Inf Dis 2008;197:1713-6. [PubMed: 18419343]

[41]. Rothfuchs A, Bafica A, Feng C, Egen J, Williams D, Brown G, et al. Dectin-1 interaction with Mycobacterium tuberculosis leads to enhanced IL-12p40 production by splenic dendritic cells. J Immunol 2007;179:3463-71. [PubMed: 17785780]

[42]. Ishikawa E, Ishikawa T, Morita Y, Toyonaga K, Yamada H, Takeuchi O, et al. Direct recognition of the mycobacterial glycolipid, trehalose dimycolate, by C-type lectin Mincle. J Exp Med 2009;206:2879-88. [PubMed: 20008526]

[43]. Schoenen H, Bodendorfer B, Hitchens K, Manzanero S, Werninghaus K, Nimmerjahn F, et al. Cutting edge: Mincle is essential for recognition and adjuvanticity of the mycobacterial cord factor and its synthetic analog trehalose-dibehenate. J Immunol 2010;184:2756-60. [PubMed: 20164423]

[44]. Werninghaus K, Babiak A, Gross O, Hölscher C, Dietrich H, Agger E, et al. Adjuvanticity of a synthetic cord factor analogue for subunit Mycobacterium tuberculosis vaccination requires FcRgamma-Syk-Card9-dependent innate immune activation. J Exp Med 2009;206:89-97. [PubMed: 19139169]

[45]. Reynolds JM, Pappu BP, Peng J, Martinez GJ, Zhang Y, Chung Y, et al. Toll-like receptor 2 signaling in $\mathrm{CD} 4(+) \mathrm{T}$ lymphocytes promotes $\mathrm{T}$ helper 17 responses and regulates the pathogenesis of autoimmune disease. Immunity 2010;32:692-702. [PubMed: 20434372]

[46]. Schulz SM, Kohler G, Holscher C, Iwakura Y, Alber G. IL-17A is produced by Th17, gammadelta T cells and other CD4- lymphocytes during infection with Salmonella enterica serovar Enteritidis and has a mild effect in bacterial clearance. Int Immunol 2008;20:1129-38. [PubMed: 18599501]

[47]. Hamada S, Umemura M, Shiono T, Tanaka K, Yahagi A, Begum MD, et al. IL-17A produced by gammadelta $\mathrm{T}$ cells plays a critical role in innate immunity against Listeria monocytogenes infection in the liver. J Immunol 2008;181:3456-63. [PubMed: 18714018]

[48]. Aujla S, Dubin P, Kolls J. Th17 cells and mucosal host defense. Semin Immunol 2007;19:37782. [PubMed: 18054248]

[49]. Okamoto Yoshida Y, Umemura M, Yahagi A, O’Brien R, Ikuta K, Kishihara K, et al. Essential role of IL-17A in the formation of a mycobacterial Infection-induced granuloma in the lung. J Immunol 2010;184:4414-22. [PubMed: 20212094]

[50]. Lockhart E, Green A, Flynn J. IL-17 production is dominated by gammadelta T cells rather than CD4 T cells during Mycobacterium tuberculosis infection. J Immunol 2006;177:4662-69. [PubMed: 16982905]

[51]. Umemura M, Yahagi A, Hamada S, Begum M, Watanabe H, Kawakami K, et al. IL-17-mediated regulation of innate and acquired immune response against pulmonary Mycobacterium bovis bacille Calmette-Guerin Infection. J Immunol 2007;178:3786-96. [PubMed: 17339477]

[52]. Sutton CE, Lalor SJ, Sweeney CM, Brereton CF, Lavelle EC, Mills KH. Interleukin-1 and IL-23 induce innate IL-17 production from gammadelta T cells, amplifying Th17 responses and autoimmunity. Immunity 2009;31:331-41. [PubMed: 19682929]

[53]. Seiler P, Aichele P, Bandermann S, Hauser A, Lu B, Gerard N, et al. Early granuloma formation after aerosol Mycobacterium tuberculosis infection is regulated by neutrophils via CXCR3signaling chemokines. Eur J Immunol 2003;33:2676-86. [PubMed: 14515251]

[54]. Silva MT. When two is better than one: macrophages and neutrophils work in concert in innate immunity as complementary and cooperative partners of a myeloid phagocyte system. $\mathbf{J}$ Leukocyte Biol 2010;87:93-106. [PubMed: 20052802]

[55]. Dragon S, Saffar A, Shan L, Gounni A. IL-17 attenuates the anti-apoptotic effects of GM-CSF in human neutrophils. Mol Immunol 2007;45:160-68. [PubMed: 17555818]

[56]. Zelante T, De Luca A, Bonifazi P, Montagnoli C, Bozza S, Moretti S, et al. IL-23 and the Th17 pathway promote inflammation and impair antifungal immune resistance. Eur J Immunol 2007;37:2695-706. [PubMed: 17899546] 
[57]. Redford P, Boonstra A, Read S, Pitt J, Graham C, Stavropoulos E, et al. Enhanced protection to Mycobacterium tuberculosis infection in IL-10-deficient mice is accompanied by early and enhanced Th1 responses in the lung. Eur J Immunol 2010;40:2200-10. [PubMed: 20518032]

[58]. Tsai M, Chakravarty S, Zhu G, Xu J, Tanaka K, Koch C, et al. Characterization of the tuberculous granuloma in murine and human lungs: cellular composition and relative tissue oxygen tension. Cell Microbiol 2006;8:218-32. [PubMed: 16441433]

[59]. D’Souza CD, Cooper AM, Frank AA, Mazzaccaro RJ, Bloom BR, Orme IM. An antiinflammatory role for gd T lymphocytes in acquired immunity to Mycobacterium tuberculosis. $\mathrm{J}$ Immunol 1997;158:1217-21. [PubMed: 9013962]

[60]. Wozniak T, Ryan A, Triccas J, Britton W. Plasmid interleukin-23 (IL-23), but not plasmid IL-27, enhances the protective efficacy of a DNA vaccine against Mycobacterium tuberculosis infection. Infect Immun 2006;74:557-65. [PubMed: 16369012]

[61]. Pedrosa J, Saunders BM, Appelberg R, Orme IM, Silva MT, Cooper AM. Neutrophils play a protective, non-phagocytic, role in systemic Mycobacterium tuberculosis infection of mice. Infect Immun 2000;68:577-83. [PubMed: 10639420]

[62]. Silva MT. Neutrophils and macrophages work in concert as inducers and effectors of adaptive immunity against extracellular and intracellular microbial pathogens. J Leukocyte Biol 2010;87:805-13. [PubMed: 20110444]

[63]. Zenewicz LA, Flavell RA. IL-22 and inflammation: leukin' through a glass onion. Eur J Immunol 2008;38:3265-8. [PubMed: 19016525]

[64]. Aujla SJ, Chan YR, Zheng M, Fei M, Askew DJ, Pociask DA, et al. IL-22 mediates mucosal host defense against Gram-negative bacterial pneumonia. Nat Med 2008;14:275-81. [PubMed: 18264110]

[65]. Scriba TJ, Kalsdorf B, Abrahams DA, Isaacs F, Hofmeister J, Black G, et al. Distinct, specific IL-17- and IL-22-producing CD4+ T cell subsets contribute to the human anti-mycobacterial immune response. J Immunol 2008;180:1962-70. [PubMed: 18209095]

[66]. Wilson MS, Feng CG, Barber DL, Yarovinsky F, Cheever AW, Sher A, et al. Redundant and pathogenic roles for IL-22 in mycobacterial, protozoan, and helminth infections. J Immunol 2010;184:4378-90. [PubMed: 20220096]

[67]. Eruslanov E, Lyadova I, Kondratieva T, Majorov K, Scheglov I, Orlova M, et al. Neutrophil responses to Mycobacterium tuberculosis infection in genetically susceptible and resistant mice. Infect Immun 2005;73:1744-53. [PubMed: 15731075]

[68]. Keller C, Hoffmann R, Lang R, Brandau S, Hermann C, Ehlers S. Genetically determined susceptibility to tuberculosis in mice causally involves accelerated and enhanced recruitment of granulocytes. Infect Immun 2006;74:4295-309. [PubMed: 16790804]

[69]. Eum S, Kong J, Hong M, Lee Y, Kim J, Hwang S, et al. Neutrophils are the predominant infected phagocytic cells in the airways of patients with active pulmonary TB. Chest 2010;137:122-28. [PubMed: 19749004]

[70]. Berry MP, Graham CM, McNab FW, Xu Z, Bloch SA, Oni T, et al. An interferon-inducible neutrophil-driven blood transcriptional signature in human tuberculosis. Nature 2010;466:973-7. [PubMed: 20725040]

[71]. Colditz G, Brewer T, Berkey C, Wilson M, Burdick E, Fineberg H, et al. Efficacy of BCG vaccine in the prevention of tuberculosis. Meta-analysis of the published literature. JAMA 1994;271:698-702. [PubMed: 8309034]

[72]. Sterne J, Rodrigues L, Guedes I. Does the efficacy of BCG decline with time since vaccination? Int J Tuberc Lung Dis 1998;2:200-07. [PubMed: 9526191]

[73]. Weir R, Gorak-Stolinska P, Floyd S, Lalor M, Stenson S, Branson K, et al. Persistence of the immune response induced by BCG vaccination. BMC Infect Dis 2008;8:9. [PubMed: 18221509]

[74]. Cruz A, Fraga A, Fountain J, Rangel-Moreno J, Torrado E, Saraiva M, et al. Pathological role of interleukin 17 in mice subjected to repeated BCG vaccination after infection with Mycobacterium tuberculosis. J Exp Med 2010;207:1609-16. [PubMed: 20624887]

[75]. Cruz A, Khader S, Torrado E, Fraga A, Pearl J, Pedrosa J, et al. Cutting edge: IFN-g regulates the induction and expansion of IL-17-producing CD4 T cells during mycobacterial infection. $\mathrm{J}$ Immunol 2006;177:1416-20. [PubMed: 16849446] 
[76]. DesVignes L, Ernst JD. Interferon- $\gamma$-responsive nonhematopoietic cells regulate the immune response to Mycobacterium tuberculosis. Immunity 2009;31:974-85. [PubMed: 20064452]

[77]. Batten M, Li J, Yi S, Kljavin N, Danilenko D, Lucas S, et al. Interleukin 27 limits autoimmune encephalomyelitis by suppressing the development of interleukin 17-producing T cells. Nat Immunol 2006;7:929-36. [PubMed: 16906167]

[78]. Stumhofer J, Laurence A, Wilson E, Huang E, Tato C, Johnson L, et al. Interleukin 27 negatively regulates the development of interleukin 17-producing $\mathrm{T}$ helper cells during chronic inflammation of the central nervous system. Nat Immunol 2006;7:937-45. [PubMed: 16906166]

[79]. Villarino A, Hibbert L, Lieberman L, Wilson E, Mak T, Yoshida H, et al. The IL-27R (WSX-1) is required to suppress T cell hyperactivity during infection. Immunity 2003;19:645-55. [PubMed: 14614852]

[80]. Pearl JE, Shabaana AK, Solache A, Gilmartin L, Ghilardi N, deSauvage F, et al. IL-27 signaling compromises control of bacterial growth in mycobacteria-infected mice. J Immunol 2004;173:7490-96. [PubMed: 15585875]

[81]. Holscher C, Holscher A, Ruckerl D, Yoshimoto T, Yoshida H, Mak T, et al. The IL-27 receptor chain WSX-1 differentially regulates antibacterial immunity and survival during experimental tuberculosis. J Immunol 2005;174:3534-44. [PubMed: 15749890]

[82]. Yoshida H, Miyazaki Y. Regulation of immune responses by interleukin-27. Immunol Rev 2008;226:234-47. [PubMed: 19161428]

[83]. McKinstry KK, Strutt TM, Buck A, Curtis JD, Dibble JP, Huston G, et al. IL-10 deficiency unleashes an influenza-specific Th17 response and enhances survival against high-dose challenge. J Immunol 2009;182:7353-63. [PubMed: 19494257]

[84]. Xu L, Kitani A, Strober W. Molecular mechanisms regulating TGF-beta-induced Foxp3 expression. Mucosal Immunol 2010;3:230-8. [PubMed: 20404810]

[85]. Scott-Browne J, Shafiani S, Tucker-Heard G, Ishida-Tsubota K, Fontenot J, Rudensky A, et al. Expansion and function of Foxp3-expressing T regulatory cells during tuberculosis. J Exp Med 2007;204:2159-69. [PubMed: 17709423]

[86]. Marin ND, Paris SC, Velez VM, Rojas CA, Rojas M, Garcia LF. Regulatory T cell frequency and modulation of IFN-gamma and IL-17 in active and latent tuberculosis. Tuberculosis (Edinb) 2010;90:252-61. [PubMed: 20594914]

[87]. Garg A, Barnes P, Roy S, Quiroga M, Wu S, García V, et al. Mannose-capped lipoarabinomannan- and prostaglandin E2-dependent expansion of regulatory T cells in human Mycobacterium tuberculosis infection. Eur J Immunol 2008;38:459-69. [PubMed: 18203140]

[88]. Shafiani S, Tucker-Heard G, Kariyone A, Takatsu K, Urdahl KB. Pathogen-specific regulatory T cells delay the arrival of effector T cells in the lung during early tuberculosis. J Exp Med 2010;207:1409-20. [PubMed: 20547826]

[89]. Afzali B, Mitchell P, Lechler RI, John S, Lombardi G. Translational mini-review series on Th17 cells: induction of interleukin-17 production by regulatory T cells. Clin Exp Immunol 2010;159:120-30. [PubMed: 19912251]

[90]. Koch M, Tucker-Heard G, Perdue N, Killebrew J, Urdahl K, Campbell D. The transcription factor T-bet controls regulatory $\mathrm{T}$ cell homeostasis and function during type 1 inflammation. Nat Immunol 2009;10:595-602. [PubMed: 19412181]

[91]. Sullivan B, Jobe O, Lazarevic V, Vasquez K, Bronson R, Glimcher L, et al. Increased susceptibility of mice lacking T-bet to infection with Mycobacterium tuberculosis correlates with increased IL-10 and decreased IFN-gamma production. J Immunol 2005;175:4593-602. [PubMed: 16177104]

[92]. Guo S, Cobb D, Smeltz RB. T-bet inhibits the in vivo differentiation of parasite-specific CD4+ Th17 cells in a T cell-intrinsic manner. J Immunol 2009;182:6179-86. [PubMed: 19414771]

[93]. Villarino A, Huang E, Hunter C. Understanding the pro- and anti-inflammatory properties of IL-27. J Immunol 2004;173:715-20. [PubMed: 15240655] 


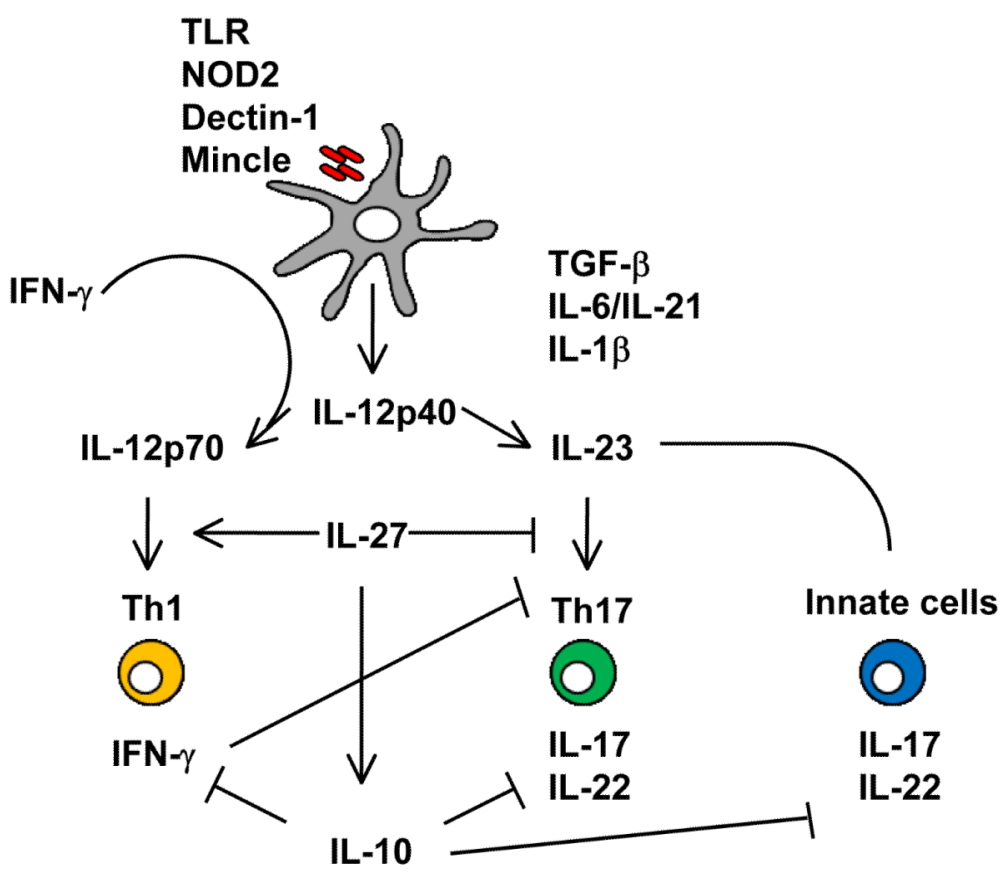

Figure 1.

Proposed model for Th1 and Th17 differentiation during TB. Upon Mtb recognition through distinct PRRs, inflammatory cytokines and IL-12p40 are expressed. In the early phases of infection IL-23 is preferentially produced which promotes innate sources of IL-17 and possibly IL-22 secretion. As the infection progress, innate cells produce IFN- $\gamma$ which enhances IL-12p35 expression and balances IL-12p70 and IL-23 production. IL-12p70 signaling on primed T cells leads to Th1 differentiation whereas TGF- $\beta$, IL- 6 and IL-23 induce Th17 differentiation. IL-27 is also induced during infection and acts on T cells to enhance T-bet expression and IFN- $\gamma$ production. IL-27 and IFN- $\gamma$ inhibit Th17 development whereas IL-10 induced dependently and independently of IL-27 regulates both IFN- $\gamma$ and IL-17 responses. 


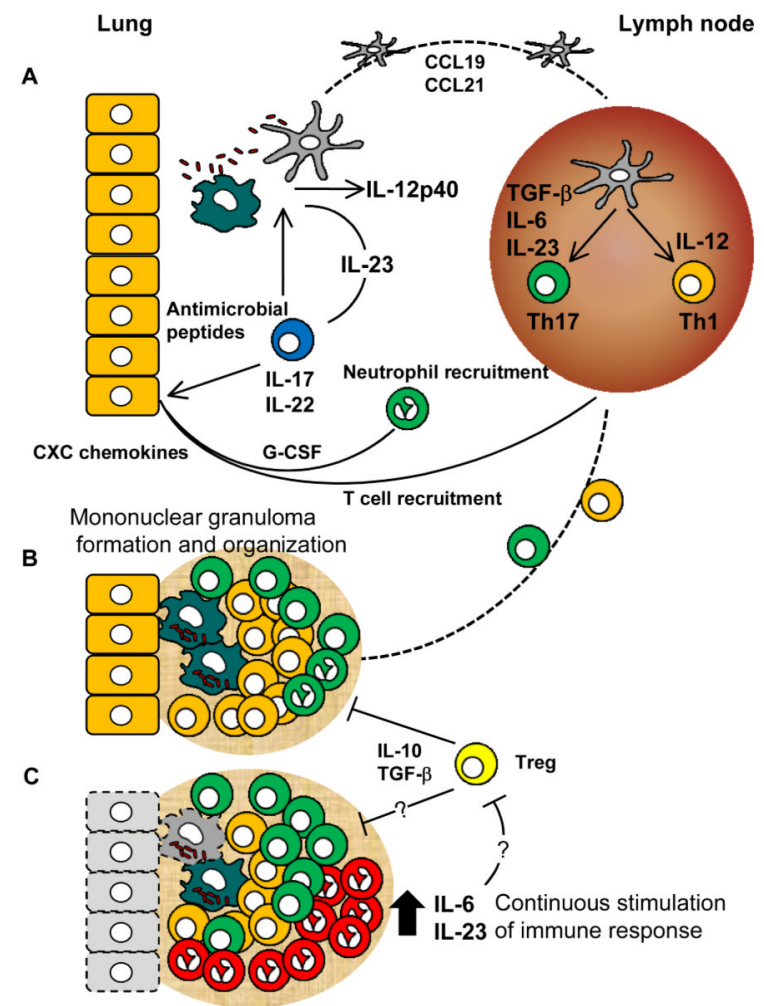

Figure 2.

IL-17 and Th17 cells play roles throughout Mtb infection. (A) During the initial stages of infection IL-17, likely produced by innate cells, acts on hematopoietic and nonhematopoietic cells promoting secretion of antimicrobial peptides, G-CSF and CXC chemokines. As DCs migrate to the lymph node, both Th1 and Th17 cell are differentiated. (B) Chemokine gradients in the infected lung promote recruitment of protective cells and a mononuclear granuloma is formed. (C) In settings where IL-23 and IL-6 are highly expressed IL-17producing cells accumulate in high numbers in the lung and immunopathological consequences develop. 